This item was submitted to Loughborough's Research Repository by the author.

Items in Figshare are protected by copyright, with all rights reserved, unless otherwise indicated.

\title{
Radiation characteristics of a conical monopole antenna with a partially corrugated ground plane.
}

PLEASE CITE THE PUBLISHED VERSION

PUBLISHER

(c) IEEE

VERSION

VoR (Version of Record)

LICENCE

CC BY-NC-ND 4.0

\section{REPOSITORY RECORD}

Mulenga, Charity B., and James A. Flint. 2019. "Radiation Characteristics of a Conical Monopole Antenna with a Partially Corrugated Ground Plane.”. figshare. https://hdl.handle.net/2134/6147. 
This item was submitted to Loughborough's Institutional Repository (https://dspace.lboro.ac.uk/) by the author and is made available under the following Creative Commons Licence conditions.

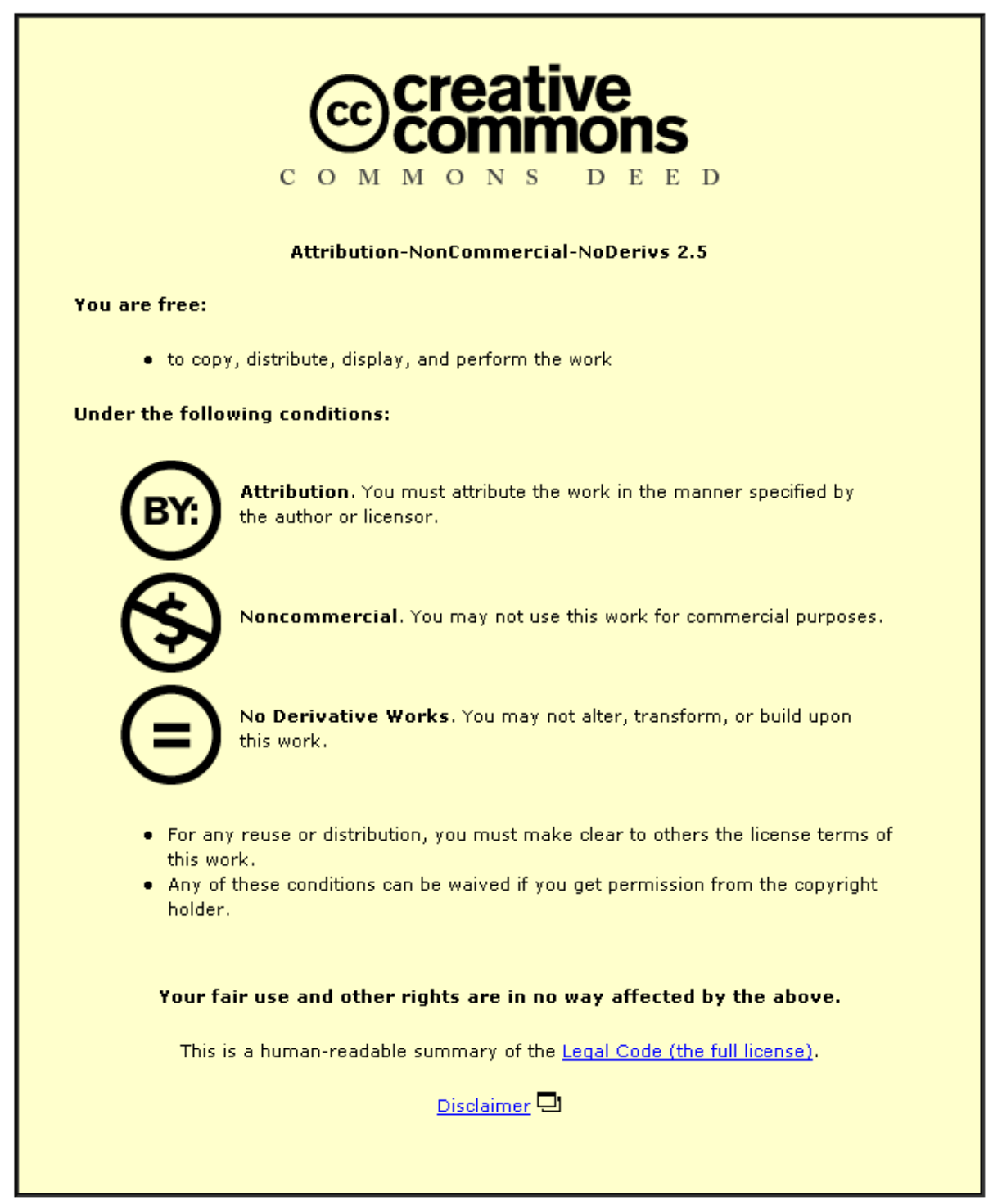

For the full text of this licence, please go to: http://creativecommons.org/licenses/by-nc-nd/2.5/ 


\title{
Radiation Characteristics of a Conical Monopole Antenna with a Partially Corrugated Ground Plane
}

\author{
Charity B. Mulenga ${ }^{1}$, James A. Flint ${ }^{2}$ \\ Electronic and Electrical Engineering Department, Loughborough University \\ Loughborough, Leicestershire, LE11 3TU, United Kingdom \\ 'C.B.Mulenga@lboro.ac.uk \\ james.flint@ieee.org
}

\begin{abstract}
In order to preserve broadband performance, the conical monopole antenna is ideally mounted on a semi-infinite ground plane. Practical finite ground planes can introduce unwanted effects in the radiation patterns such as significant back lobes. Periodic surfaces such as rotationally symmetric corrugated structures have been proven capable of improving the radiation characteristics of a variety of antennas. In this paper, the radiation patterns of a conical antenna with a PEC reflector are initially presented. The $P E C$ reflector is then replaced with a partially corrugated reflector surface. The effects of the partially corrugated reflector on the radiation patterns and electric field distribution along the reflector surface are investigated. The simulation results reveal that the radiation patterns of a conical antenna with a partially corrugated reflector are more stable over a wide frequency band than that for the $\mathrm{PEC}$ reflector.
\end{abstract}

\section{INTRODUCTION}

Finite ground planes for monopole antennas can be viewed as having two main functional properties. Firstly they act as a plane of symmetry which in the ideal case allows the device to produce a radiation pattern that is identical to the equivalent dipole over one hemisphere. Secondly, the presence of the ground plane provides shielding in the other hemisphere. In certain applications it is highly desirable to mitigate the effects of finite (and imperfect) ground planes. The objective of the current paper is to investigate whether applying surface wave modification techniques such as corrugated grounds can achieve this objective.

The vast potential of periodic structures in improving the performance of antennas has been highlighted by a variety of researchers from the optical, electromagnetics and antenna research domains [1-6]. Periodic structures have been extensively used to suppress the propagation of surface waves within a known frequency band. The control of such waves has been used to reduce mutual coupling in antenna arrays [7], increase the gain of various antennas [8,9] and for antenna radiation pattern improvements $[10,11]$. There exist many different types of periodic structures which can offer the required control of surface waves, from the typical EBGs and photonic crystals to anisotropic surfaces such as soft and hard surfaces [12]. The main effects of a finite ground are the introduction of standing waves between the antenna feed point and the edge and also the presence of diffraction due to the discontinuity. In each case it seems reasonable to believe that applying techniques such as electromagnetic band gap (EBG) [13] could have a useful effect on the surface currents and consequently on reducing the effects of the truncation.

Rotationally symmetrical antennas lend themselves towards rotationally symmetrical periodic surfaces. The advantage of these periodic surfaces is that a signal generated by a source located at the centre of the reflector experiences identical bandgap effects in all radial directions. Llombart et al. [14] proposed the use of planar circularly symmetric periodic structures for enhancing the performance of planar printed, slot-coupled dipole antennas. Here, the periodic structural elements were arranged in a circular symmetric form round the central antenna in order to impede the propagation of $\mathrm{TM}_{0}$ surface waves in planar printed structures. Further examples of the application of rotationally symmetric periodic structures can be found in $[8,10,15]$.

In this paper, the concept of circularly symmetric corrugated structures is introduced to improve the radiation characteristics of conical antennas. The proposed corrugated structures are based on the rotationally symmetric corrugations with a rectangular cross section. There exist many ways by which soft surfaces can be realized. For example dielectric loading, or strip loaded dielectrics [16]. However, in this study the alternative approach of using quarter wavelength deep choke rings will be followed.

This paper is organized as follows: Section II presents the radiation characteristics of a conical antenna backed by a PEC reflector. In Section III the PEC reflector is modified to a partially corrugated reflector. The radiation characteristics of the conical antenna above the corrugated reflector are analyzed and discussed. And finally conclusions are drawn in Section IV. Throughout this paper, the analysis is performed primarily using a TLM based technique [17].

\section{CONICAL ANTENNA ABOVE A FLAT FINITE PEC REFLECTOR}

Fig. 1 shows a hemispherical-capped conical antenna mounted on a circular finite PEC reflector of radius $r$. The conical antenna is designed to operate in the band $3-15 \mathrm{GHz}$, 
which results in a height $h=0.25 \lambda_{\mathrm{LF}}$, and a top circular diameter $a=0.5 \lambda_{\mathrm{LF}}$, where $\lambda_{\mathrm{LF}}$ is the wavelength at the design frequency $3 \mathrm{GHz}$. A cone half angle of $\theta_{o}=45^{\circ}$ is chosen and the antenna is fed by a standard $50-\Omega$ coaxial cable. Fig. 2 shows the radiation patterns of the PEC conical antenna for a range of frequencies across the entire band for two reflector radii.

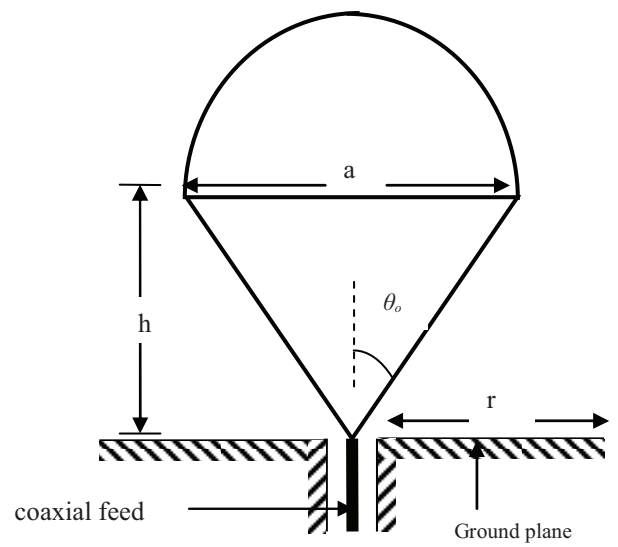

Fig. 1 Conical antenna at the centre of a circular ground plane of radius $r$

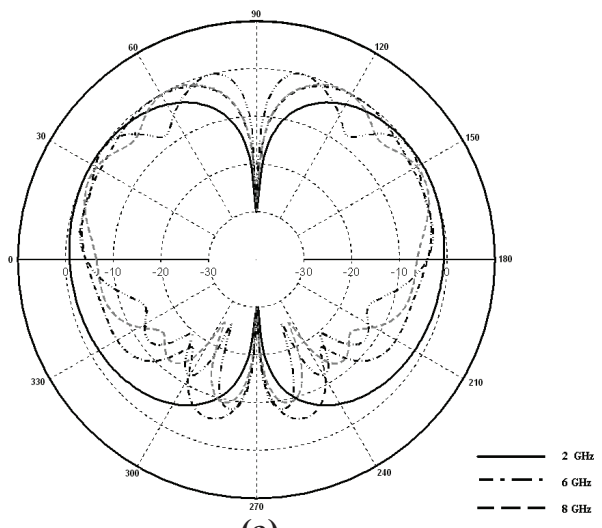

(a)

$---{ }^{8 \mathrm{GH} x}$
$-\cdots-{ }_{10 \mathrm{GH}}$

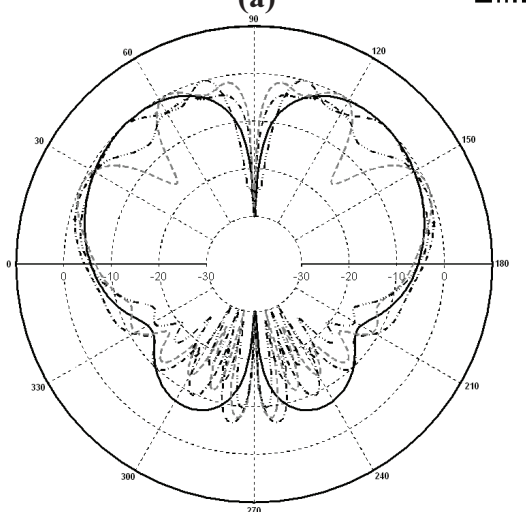

(b)

Fig. 2: Radiation patterns of a PEC conical antenna for different ground plane radii. (a) $r=1.67 \lambda_{3 \mathrm{GHz}}$, and (b) $r=3.33 \lambda_{3 \mathrm{GHz}}$.
At low frequencies, the antenna radiation patterns are primarily dipole-like, showing distortion for the larger ground plane size. It is also evident that the overall pattern asymmetry increases with frequency. The approach undertaken in this paper to reduce the back radiation is to introduce a set of corrugations into a portion of the reflector. This forms the discussion of the subsequent section.

\section{CONICAL ANTENNA ABOVE A ROTATIONALLY SYMMETRIC CORRUGATED REFLECTOR}

A schematic diagram of a conical antenna (not to scale) with a coaxially corrugated reflector is shown in Fig. 3. The corrugated reflector structure consists of circular ridges and slots cut into the metal slab, whose top surface coincides with the $x-y$ plane of the rectangular coordinate system. The corrugated structure forms a rotationally symmetric cylindrical corrugated structure designed to operate at $f_{o}=$ $10 \mathrm{GHz}\left(\lambda_{c}=30 \mathrm{~mm}\right)$. The important geometric parameters for the 'soft' reflector are the tooth width, $t$, gap width, $g$, corrugation depth, $d$ reflector diameter, $2 r$, and reflector thickness, $T_{k}$. The design of the corrugated surface follows the method outlined in [16]. Typical values for these parameters are given in Fig. 3. Note that the conical antenna remains unchanged, having the configuration parameters specified as in Section II.

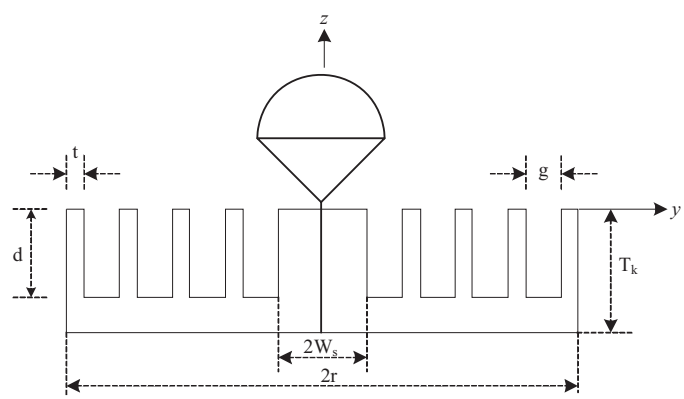

(a)

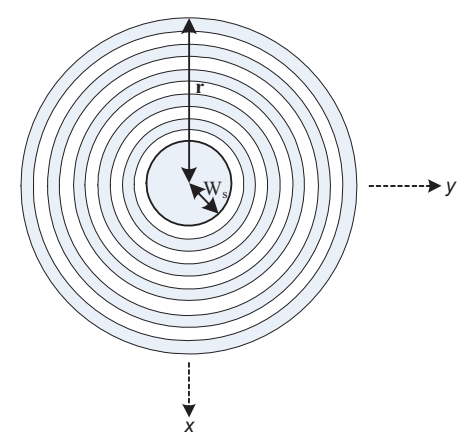

(b)

Fig. 3: Cross section of a conical antenna backed by a flat corrugated ground plane (a) side view (b) top View. Design parameters are: $d=0.25 \lambda_{\mathrm{o}}, t=$ $0.03 \lambda_{0}, g=0.67 \lambda_{0}, T_{k}=0.27 \lambda_{0}, r=2.67 \lambda_{0}$ and $W_{s}=1.67 \lambda_{0}$ 


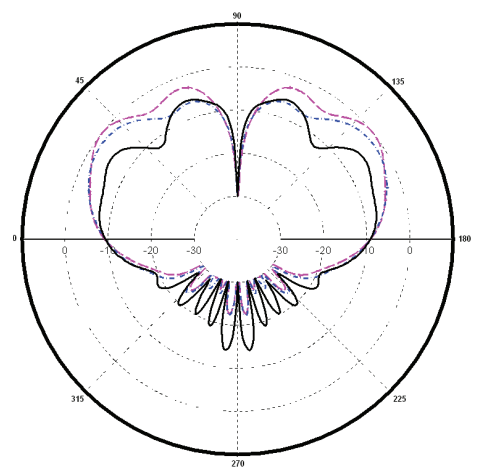

(a)

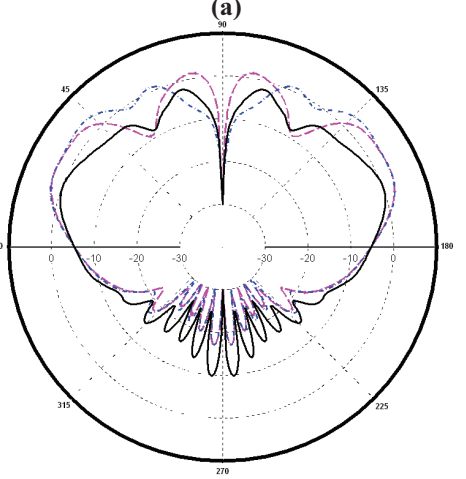

(b)

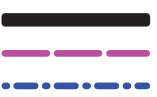

PEC

Corrugated ground plane (10 corrugations) Corrugated ground plane (15 corrugations)

Fig. 4: Radiation pattern for a soft conical antenna, where the corrugation depth $d=0.25 \lambda_{o}, t=0.03 \lambda_{o}, g=0.67 \lambda_{o}, T_{k}=0.27 \lambda_{o}, r=2.67 \lambda_{o}$ and $W_{s}=1.67 \lambda_{o}$ (10 corrugations) and $1.17 \lambda_{o}$ (15 corrugations) for (a) $f_{o}$ (b) $1.2 f_{o}$

Following various optimization studies carried out in this work, the corrugations were chosen to start at a distance $W_{s}=1.67 \lambda_{c}$ from the center of the reflector. This optimized distance was selected such that: 1) the bandwidth of the antenna structure remains the same as that established in Section II (2) corrugations do not exist within the antenna's spherical radius which is equal to the antenna side length $\left(h \sin \theta_{o}\right)$. The radiation patterns of conical antennas backed by partially corrugated reflectors with two different corrugation periodicities are investigated. The simulated radiation pattern results are shown in Fig. 4. As evidently visible, the corrugated reflector reduces the back radiation and the pattern above horizon develops into a more symmetrical dipole-like pattern. The backward radiation is directly related to the currents induced over the ground plane. The surface currents on the partially corrugated reflector are concentrated round the conical antenna. The corrugations suppress the surface currents flowing towards the edges of the reflector, resulting in a reduction in backward radiation. This is clearly visible from the E-field plots in Fig. 5. Ultimately, the radiation is confined in the upper hemisphere with very little field being diffracted to the back face of the ground plane.

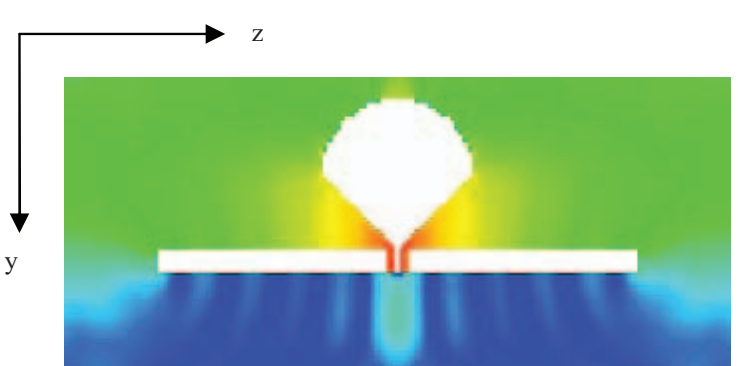

(a)

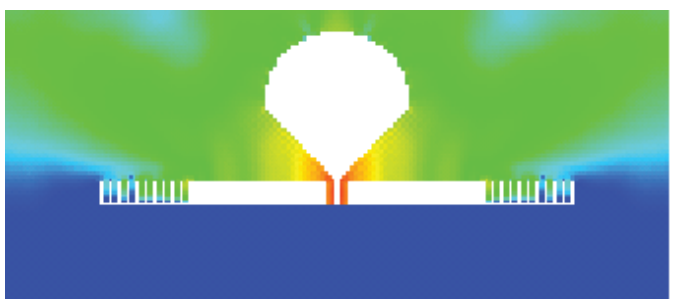

(b)

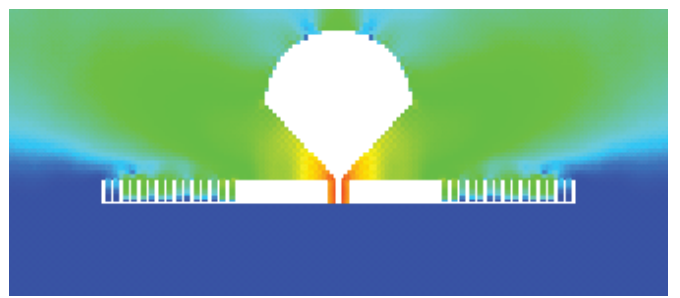

(c)

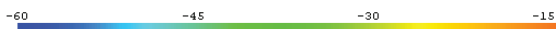

$(\mathrm{dB})$

Fig. 5: Free space normalized E-field distribution z-y cut away plane at $10 \mathrm{GHz}$. The E-field values are referenced to the maximum field strength in the simulation. (a) PEC reflector (b) Corrugated reflector (10 slots) (c) Corrugated reflector (15 slots)

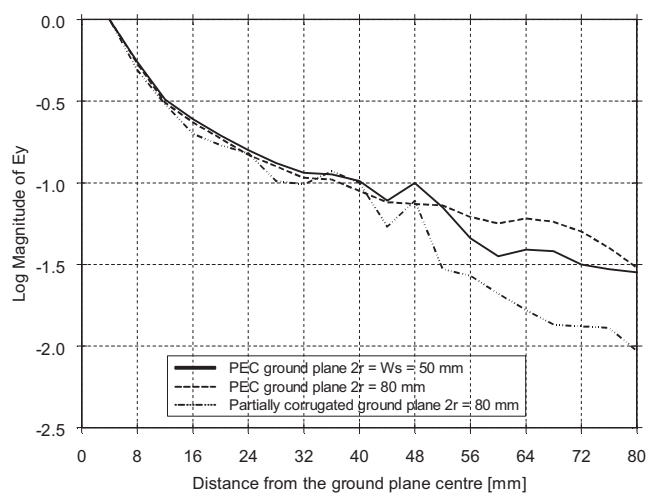

Fig. 6: Simulated normalized Log magnitude $\left|E_{y}\right|$ as a function of radial distance from the feed point. The field was extracted from the simulation at a distance of $0.05 \mathrm{~mm}$ above the antenna ground plane. 


\section{ANALYSIS OF THE FIELD AT THE INTERFACE BETWEEN AIR AND THE GROUND PLANE}

Here, a TLM code has been used to compute the distribution or decay rate of the transverse electric field at the air-ground plane interface for the conical antenna with and without the partially corrugated reflector. Fig. 6 shows the distribution of the transverse field at the surface of the ground plane of the conical antenna for (a) ground plane with radius $2 r=W_{s}=50 \mathrm{~mm}$ (b) ground plane with radius $2 r=80 \mathrm{~mm}$ and (c) corrugated ground plane with radius $2 \mathrm{r}=80 \mathrm{~mm}$ and $W_{s}=50 \mathrm{~mm}$. The decay rate of the fields was evaluated by placing observation points $0.05 \mathrm{~mm}$ above the ground plane. From Fig. 6, the following observations can be made about the decay rate of the fields:

- Assuming that the surface wave is dominant for PEC reflectors, it is found that the electric fields decay as

$$
E_{y} \propto \frac{1}{r}
$$

where $r$ is the horizontal distance away from the axis of the antenna.

- For the partially corrugated ground plane, the electric fields decay as follows

$$
E_{y} \propto \frac{1}{r^{2}}
$$

The decay rate is similar to that for lateral fields which decay as $1 / \mathrm{r}^{2}$ [8]. From this, it can be concluded that the corrugated reflector suppresses the propagation of surface waves.

\section{CONCLUSIONS}

In this paper, the radiation characteristics of a conical antenna with and without a partially corrugated reflector have been presented. The simulated radiation patterns demonstrate that partially corrugating the reflector reduces the back radiation and enhances the forward radiation through the suppression of surface wave currents. Comparisons between the decay rate of the normal electric field the surface of the reflectors suggest that when a corrugated reflector is employed, the magnitude of the surface current at the edges is reduced. Consequently, the effects edge diffraction can potentially be mitigated by this technique.

\section{REFERENCES}

[1] L. Zhang, N. G. Alexopoulos, D. F. Sievenpiper and E. Yablonovitch, "An efficient finite-element method for the analysis of photonic band- gap materials," in Microwave Symposium Digest, 1999 IEEE MTT-S International, 1999, pp. 1703-1706 vol.4.

[2] D. Maystre, "Electromagnetic study of photonic band gaps," Pure and Applied Optics, vol. 3, pp. 975-993, 1994.

[3] Y. L. R. Lee, A. Chauraya, D. S. Lockyer and J. C. Vardaxoglou, "Dipole and tripole metallodielectric photonic bandgap (MPBG) structures for microwave filter and antenna applications," Optoelectronics, IEE Proceedings -, vol. 147, pp. 395-400, 2000.

[4] Y. Rahmat-Samii and H. Mosallaei, "Electromagnetic band-gap structures: Classification, characterization, and applications," in Antennas and Propagation, 2001.Eleventh International Conference on (IEE Conf.Publ.no.480), 2001, pp. 560-564 vol.2.

[5] F. Yang and Y. Rahmat-Samii, "Microstrip antennas integrated with electromagnetic band-gap (EBG) structures: a low mutual coupling design for array applications," Antennas and Propagation, IEEE Transactions on, vol. 51, pp. 2936-2946, 2003.

[6] R. Abhari and G. V. Eleftheriades, "Metallo-dielectric electromagnetic bandgap structures for suppression and isolation of the parallel-plate noise in high-speed circuits," Microwave Theory and Techniques, IEEE Transactions on, vol. 51, pp. 1629-1639, 2003.

[7] Fan Yang and Y. Rahmat-Samii, "Microstrip antennas integrated with electromagnetic band-gap (EBG) structures: a low mutual coupling design for array applications," IEEE Trans. Antennas and Propagat., vol. 51, pp. 2936-2946, 2003.

[8] H. Boutayeb and T. A. Denidni, "Gain Enhancement of a Microstrip Patch Antenna Using a Cylindrical Electromagnetic Crystal Substrate," IEEE Trans. Antennas and Propagat., vol. 55, pp. 3140-3145, 2007.

[9] N. Llombart, P. de Maagt, A. Neto and G. Gerini, "Planar circularly symmetric EBG structures for reducing surface waves in printed antennas," IEEE Trans. Antennas and Propagat., vol. 53, pp. 3210$3218,2005$.

[10] H. Nakano, K. Hitosugi, N. Tatsuzawa, D. Togashi, H. Mimaki and J. Yamauchi, "Effects on the radiation characteristics of using a corrugated reflector with a helical antenna and an electromagnetic band-gap reflector with a spiral antenna," Antennas and Propagation, IEEE Transactions on, vol. 53, pp. 191-199, 2005.

[11] V. Rumsey, "Horn antennas with uniform power patterns around their axes," Antennas and Propagation, IEEE Transactions on, vol. 14, pp. 656-658, 1966.

[12] P. -. Kildal, "Definition of artificially soft and hard surfaces for electromagnetic waves," Electronics Letters, vol. 24, pp. 168-170, 1988.

[13] D. Sievenpiper, Lijun Zhang, R. F. J. Broas, N. G. Alexopolous and E. Yablonovitch, "High-impedance electromagnetic surfaces with a forbidden frequency band," Microwave Theory and Techniques, IEEE Transactions on, vol. 47, pp. 2059-2074, 1999.

[14] N. Llombart, A. Neto, P. De Maagt and G. Gerini, "Bandwidth, efficiency and directivity enhancement of printed antenna performance using planar circularly symmetric EBGs," 35th European Microwave Conference 2005 - Conference Proceedings, vol. 3, pp. 1859-1862, 2005.

[15] Z. Ying and P. S. Kildal, "Improvements of dipole, helix, spiral, microstrip patch and aperture antennas with ground planes by using corrugated soft surfaces," Microwaves, Antennas and Propagation, IEE Proceedings -, vol. 143, pp. 244-248, 1996.

[16] Zhinong Ying, "Study of different realizations and calculation models for soft surfaces by using a vertical monopole on a soft disk as a test bed," Antennas and Propagation, IEEE Transactions on, vol. 44, pp. 1474-1481, 1996

[17] P. B. Johns, "A Symmetrical Condensed Node for the TLM Method," Microwave Theory and Techniques, IEEE Transactions on, vol. 35, pp. 370-377, 1987. 